University of New Orleans

ScholarWorks@UNO

$9-1982$

\title{
Stationary property of normal-incidence reflection from isotropic surfaces
}

R. M.A. Azzam

University of New Orleans, razzam@uno.edu

Follow this and additional works at: https://scholarworks.uno.edu/ee_facpubs

Part of the Electrical and Electronics Commons, and the Physics Commons

\section{Recommended Citation}

R. M. A. Azzam, "Stationary property of normal-incidence reflection from isotropic surfaces," J. Opt. Soc. Am. 72, 1187-1189 (1982)

This Article is brought to you for free and open access by the Department of Electrical Engineering at ScholarWorks@UNO. It has been accepted for inclusion in Electrical Engineering Faculty Publications by an authorized administrator of ScholarWorks@UNO. For more information, please contact scholarworks@uno.edu. 


\title{
Stationary property of normal-incidence reflection from isotropic surfaces
}

\author{
R. M. A. Azzam \\ Department of Electrical Engineering, University of New Orleans, Lakefront, New Orleans, Louisiana 70148
}

Received March 18, 1982; revised manuscript received April 26, 1982

\begin{abstract}
The complex reflection coefficients for the parallel $(p)$ and perpendicular $(s)$ polarizations of light that are normally incident upon an isotropic surface are proved to be stationary with respect to small changes of the angle of incidence in the neighborhood of zero. This is true not only for a single interface between isotropic media but also for any one-dimensionally inhomogeneous or multilayer reflecting structure that is stratified in the direction of the surface normal. For incident light of certain intensity, phase, and polarization, the intensity, phase, and polarization of the reflected light all remain stationary with respect to small near-normal angle-of-incidence variations to first order. A second-order analysis is carried out to determine the parabolic (quadratic) variation of various reflection parameters of an interface with an angle near normal incidence.
\end{abstract}

\section{INTRODUCTION}

An established method for the determination of optical properties (complex refractive index or complex dielectric function) of materials (absorbing solids or liquids) is based on reflectance measured at normal incidence, usually as a function of incident photon energy or wavelength. ${ }^{1}$ In carrying out such measurements, normal incidence is often loosely interpreted to mean incidence at angles within $\sim 15^{\circ}$ to the surface normal. The assumption is that Fresnel's reflection coefficients are stationary with respect to small angle-of-incidence variations near normal incidence. In this paper we prove that this stationary property indeed holds and that it is more general than is commonly thought. We find that it applies to (1) both the parallel $(p)$ and perpendicular (s) polarizations (hence to all incident polarizations), (2) the magnitude and angle of the reflection coefficients (hence to the amplitude and phase of the reflected wave), and (3) a single interface as well as to multilayer or one-dimensional continuously inhomogeneous structures. Furthermore, the quadratic variations of reflection parameters of an interface with angle near normal incidence are determined.

\section{REFLECTION BY A SINGLE INTERFACE}

For a planar interface between two homogeneous and isotropic media of dielectric functions $\epsilon_{0}$ and $\epsilon_{1}$, Fresnel's complex amplitude-reflection coefficients for the $s$ and $p$ polarizations at an angle of incidence $\phi$ (in medium 0 ) are given by ${ }^{2}$

$$
\begin{aligned}
& r_{01 s}=\left(S_{0}-S_{1}\right) /\left(S_{0}+S_{1}\right), \\
& r_{01 p}=\left(\epsilon_{1} S_{0}-\epsilon_{0} S_{1}\right) /\left(\epsilon_{1} S_{0}+\epsilon_{0} S_{1}\right),
\end{aligned}
$$

where

$$
S_{i}=\left(\epsilon_{i}-\epsilon_{0} \sin ^{2} \phi\right)^{1 / 2}, \quad i=0,1 .
$$

If we take the first derivative with respect to $\phi$ (to be indicated throughout by a prime superscript) of Eqs. (1)-(3), we get

$$
r_{01 s}{ }^{\prime}=2\left(S_{0}{ }^{\prime} S_{1}-S_{0} S_{1}{ }^{\prime}\right) /\left(S_{0}+S_{1}\right)^{2}
$$

$$
\begin{aligned}
r_{01 p}{ }^{\prime} & =2 \epsilon_{0} \epsilon_{1}\left(S_{0}{ }^{\prime} S_{1}-S_{0} S_{1}{ }^{\prime}\right) /\left(\epsilon_{1} S_{0}+\epsilon_{0} S_{1}\right)^{2}, \\
S_{i}{ }^{\prime} & =a / S_{i},
\end{aligned}
$$

where

$$
a=-1 / 2 \epsilon_{0} \sin 2 \phi .
$$

At normal incidence, $\phi=0, \sin 2 \phi=0, a=0$, and $S_{i}{ }^{\prime}=0$. Consequently, from Eqs. (4) and (5), it follows also that

$$
r_{01 s}{ }^{\prime}=r_{01 p}{ }^{\prime}=0,
$$

irrespective of $\epsilon_{0}$ and $\epsilon_{1}$, i.e., for all interfaces. Equation (8) represents the stationary property of Fresnel's complex interface reflection coefficients at normal incidence.

\section{REFLECTION BY A SINGLE-LAYER- COATED OR MULTILAYER-COATED SUBSTRATE}

The complex amplitude-reflection coefficients of an all-isotropic single-layer-coated substrate are given by ${ }^{3}$

$$
\begin{aligned}
R_{\nu} & =\frac{r_{01 \nu}+r_{12 \nu} X}{1+r_{01 \nu} r_{12 \nu} X}, \quad \nu=p, s, \\
X & =\exp \left[-j 4 \pi(d / \lambda) S_{1}\right],
\end{aligned}
$$

where $r_{i j \nu}$ is Fresnel's reflection coefficient of the $i j$ interface for the $\nu$ polarization; 0,1 , and 2 represent the medium of incidence (ambient), film, and substrate, respectively; $d$ is the thickness of the homogeneous uniform layer, $\lambda$ is the wavelength; and $S_{1}$ is as previously defined in Eqs. (3).

Differentiation of Eqs. (9) with respect to $\phi$ gives

$$
\begin{aligned}
& \frac{R_{\nu}^{\prime}}{R_{\nu}}=\frac{\left(r_{01 \nu}{ }^{\prime}+r_{12 \nu}{ }^{\prime} X+r_{12 \nu} X^{\prime}\right)}{\left(r_{01 \nu}+r_{12 \nu} X\right)} \\
& -\frac{\left(r_{01 \nu}{ }^{\prime} r_{12 \nu} X+r_{01 \nu} r_{12 \nu}{ }^{\prime} X+r_{01 \nu} r_{12 \nu} X^{\prime}\right)}{\left(1+r_{01 \nu} r_{12 \nu} X\right)},
\end{aligned}
$$

where, from Eq. (10),

$$
X^{\prime}=-j 4 \pi(d / \lambda) S_{1}{ }^{\prime} X .
$$


At normal incidence, $r_{01 \nu}{ }^{\prime}=r_{12 \nu}{ }^{\prime}=0$ for $\nu=p$, s, as was proved in Section 2. Also from Eqs. (6) and (7), $S_{1}{ }^{\prime}=0$ at $\phi=0$, and that makes $X^{\prime}=0$. It follows from Eq. (11) that

$$
R_{\nu}{ }^{\prime}=0, \quad \nu=p, s,
$$

i.e., the overall (three-phase) reflection coefficients are stationary with $\phi$ at $\phi=0$.

If a second layer is added, Eqs. (9) can be amended to apply in this case by substituting for $r_{12 \nu}$ the reflection coefficient of the substructure that consists of the substrate (now medium 3 ) and the first film (medium 2). Because the latter reflection coefficient is stationary at $\phi=0$, the overall reflection coefficient for the two-layer-substrate system will be stationary. By recurrence, this argument continues to hold for any number of isotropic layers, so that Eqs. (13) are satisfied by the corresponding overall reflection coefficients.

An inhomogeneous structure that is continuously graded (in the surface-normal direction) can be approximated to any desired degree of accuracy by a stack of layers of sufficient number. It follows that the complex coefficients of reflection $R_{\nu}$ from such a structure satisfy the stationary property $R_{\nu}{ }^{\prime}$ $=0$ at $\phi=0$.

\section{DERIVED STATIONARY PROPERTIES}

As we have found, the complex reflection coefficients $R_{\nu}$ of an isotropic structure are stationary with respect to small variations of $\phi$ near $\phi=0$. This means that not only the reflectance, $\left|R_{\nu}\right|$ or $\left|R_{\nu}\right|^{2}$, but also the reflection phase shifts, $\delta_{\nu}=\arg$ $R_{\nu}$ (where $\nu=p$ or $\nu=s$ ), are stationary with respect to $\phi$ near $\phi=0$. To see this, write any complex amplitude-reflection coefficient as

$$
R_{\nu}=\left|R_{\nu}\right| e^{j \delta \nu} .
$$

Differentiation of Eq. (14) gives

$$
\frac{R_{\nu}{ }^{\prime}}{R_{\nu}}=\frac{\left|R_{\nu}\right|^{\prime}}{\left|R_{\nu}\right|}+j \delta_{\nu}^{\prime} .
$$

In Eq. (15), if we set $R_{\nu}{ }^{\prime}=0$, we get

$$
\begin{aligned}
\left|R_{\nu}\right|^{\prime} & =0, \\
\delta_{\nu}{ }^{\prime} & =0 .
\end{aligned}
$$

Equations (16) and (17) express the stationary behavior of the normal-incidence amplitude reflectance and phase shift, respectively, with respect to small $\phi$ changes. Of course, the intensity reflectance

$$
\mathscr{R}_{\nu}=\left|R_{\nu}\right|^{2}
$$

is also stationary because

$$
\mathcal{R}_{\nu}{ }^{\prime}=2\left|R_{\nu} \| R_{\nu}\right|^{\prime}=0
$$

when $\left|R_{\nu}\right|^{\prime}=0$.

Also, the (ellipsometric) ratio of $p$ - and $s$-reflection coefficients (hence the state of polarization of the reflected light for a given incident polarization) is stationary with respect to small $\phi$ changes near $\phi=0$. Thus if we write

$$
\rho=R_{p} / R_{s},
$$

then

$$
\rho^{\prime}=\left(R_{p}{ }^{\prime} / R_{s}\right)-\left(R_{p} R_{s}{ }^{\prime} / R_{s}{ }^{2}\right)
$$

and

$$
\rho^{\prime}=0
$$

when $R_{p}{ }^{\prime}=R_{s}{ }^{\prime}=0$ at $\phi=0$.

If an angle-of-incidence derivative ellipsometry and reflectometry experiment ${ }^{4,5}$ is carried out at normal incidence, all derivative signals will disappear to first order in the angle-of-incidence excursion $\Delta \phi$.

\section{ANISOTROPIC SURFACES}

Although details are not included here, it can be proved 6 that the stationary property of normal-incidence reflection extends to uniaxial anisotropic stratified structures in which the optic axis coincides with the direction of stratification and is normal to the surface. We expect that such a property will also continue to hold for other symmetric anisotropies; however, further generalization cannot be made at this point.

\section{SECOND DERIVATIVES FOR A SINGLE INTERFACE}

Because the first derivatives with respect to $\phi$ of Fresnel's interface reflection coefficients are zero at $\phi=0$, the change of these coefficients, as one goes off normal incidence by a small angle, is determined by the next nonvanishing higherorder derivative. Differentiation of Eqs. (4) and (5) gives the following second derivatives that are valid at any $\phi$ :

$$
\begin{aligned}
r_{01 s^{\prime \prime}}= & {\left[2\left(S_{0}+S_{1}\right)\left(S_{0}{ }^{\prime \prime} S_{1}-S_{0} S_{1}{ }^{\prime \prime}\right)\right.} \\
& \left.-4\left(S_{0}{ }^{\prime}+S_{1}\right)\left(S_{0}{ }^{\prime} S_{1}-S_{0} S_{1}{ }^{\prime}\right)\right] /\left(S_{0}+S_{1}\right)^{3}, \\
r_{01 p}{ }^{\prime \prime}= & 2 \epsilon_{0} \epsilon_{1}\left[\left(\epsilon_{1} S_{0}+\epsilon_{0} S_{1}\right)\left(S_{0}{ }^{\prime \prime} S_{1}-S_{0} S_{1}{ }^{\prime \prime}\right)\right. \\
& \left.-2\left(\epsilon_{1} S_{0}{ }^{\prime}+\epsilon_{0} S_{1}{ }^{\prime}\right)\left(S_{0}{ }^{\prime} S_{1}-S_{0} S_{1}\right)\right] /\left(\epsilon_{1} S_{0}+\epsilon_{0} S_{1}\right)^{3},
\end{aligned}
$$

where

$$
S_{i}^{\prime \prime}=\left(a^{\prime} / S_{i}\right)-\left(a^{2} / S_{i}{ }^{3}\right), \quad a^{\prime}=-\epsilon_{0} \cos 2 \phi .
$$

Evaluation of Eqs. (23) and (24) at $\phi=0$ leads to the simple result that

$$
\begin{gathered}
r_{01 s}{ }^{\prime \prime}(0)=2 N_{01} r_{01 s}(0), \\
r_{01 p}{ }^{\prime \prime}(0)=-2 N_{01} r_{01 p}(0),
\end{gathered}
$$

where

$$
N_{01}=\left(\epsilon_{0} / \epsilon_{1}\right)^{1 / 2}
$$

is the relative refractive index of the interface and the argument 0 indicates that the functions are evaluated at $\phi=0$. The difference in sign between Eqs. (26) and (27) is a consequence of our adoption of the Nebraska (Muller) conventions. ${ }^{7}$

It is now possible to write down the first three terms (the second term being zero) of a Maclaurin-series expansion,

$$
f(x)=f(0)+x f^{\prime}(0)+\frac{x^{2}}{2 !} f^{\prime \prime}(0)+\ldots,
$$

of Fresnel's interface reflection coefficients near normal incidence:

$$
\begin{aligned}
& r_{01 s}{ }^{\prime \prime}(\phi)=r_{01 s}(0)\left(1+N_{01} \phi^{2}+\ldots\right), \\
& r_{01 p}{ }^{\prime \prime}(\phi)=r_{01 p}(0)\left(1-N_{01} \phi^{2}+\ldots\right) .
\end{aligned}
$$


From Eqs. (29) and (30) the ratio of reflection coefficients $\rho$ $=r_{01 p} / r_{01 s}$ is

$$
\rho(\phi)=\rho(0)\left(1-2 N_{01} \phi^{2}+\ldots\right),
$$

where $\rho(0)=-1$ in the Nebraska (Muller) conventions.?

It is also convenient to work with the fractional changes of Fresnel's coefficients and their ratio that result from a change of angle of incidence from zero (normal incidence) to a small angle $\phi$ :

$$
\begin{aligned}
\Delta r_{01 \nu} / r_{01 \nu} & = \pm N_{01} \phi^{2}, \\
\Delta \rho / \rho & =-2 N_{01} \phi^{2} .
\end{aligned}
$$

In Eq. (32), the plus and the minus correspond to $\nu=s$ and $\nu$ $=p$, respectively. Writing $r=|r| e^{j \delta}, \rho=|\rho| e^{j \Delta}$, and taking the real and imaginary parts of Eqs. (32) and (33), we obtain

$$
\begin{aligned}
\Delta\left|r_{01 \nu}\right| / r_{01 \nu} \mid & = \pm n_{01} \phi^{2}, \\
\Delta \delta_{01 \nu} & = \pm k_{01} \phi^{2}, \\
\Delta|\rho| / \rho \mid & =-2 n_{01} \phi^{2}, \\
\Delta \Delta & =-2 k_{01} \phi^{2} .
\end{aligned}
$$

It also follows from Eqs. (18) and (34) that the fractional change of intensity reflectance is given by

$$
\Delta \mathscr{R}_{01 v} / \mathcal{R}_{01 v}= \pm 2 n_{01} \phi^{2} .
$$

In Eqs. (34), (35), and (38), the upper and lower signs correspond to $\nu=s$ and $\nu=p$, respectively:

$$
N_{01}=n_{01}+j k_{01}
$$

and

$N_{01}=1 / N_{10}=1 /\left(n_{10}-j k_{10}\right)=\left(n_{10}+j k_{10}\right) /\left(n_{10}{ }^{2}+k_{10}{ }^{2}\right)$.

When the medium of incidence is vacuum or air $\left(N_{0}=1\right), n_{10}$ and $k_{10}$ represent the refractive index and extinction coefficient of the medium of refraction, respectively. Equations (34)-(38) determine the ranges of angle of incidence over which the different reflection parameters change by specified amounts from their normal-incidence values. For instance, Eq. (38) can be solved for $\phi$ :

$$
\begin{aligned}
\phi & =|\Delta \mathcal{R} / \mathcal{R}|^{1 / 2} /\left(2 n_{01}\right)^{1 / 2} \\
& =|\Delta \mathcal{R} / \mathcal{R}|^{1 / 2}\left(n_{10}{ }^{2}+k_{10}\right)^{1 / 2} /\left(2 n_{10}\right)^{1 / 2} .
\end{aligned}
$$

For an air-glass interface, $n_{10}=1.5, k_{10}=0$, and we get $\phi=$ $5^{\circ}, 10^{\circ}$ for 1 and $4 \%$ fractional changes in reflectance, respectively. For an air-gold interface at $\lambda=0.55 \mu \mathrm{m}, n_{10}=$ $0.33, k_{10}=2.32$ (Ref. 8), and the ranges of $\phi$, calculated from Eq. (41), for $\Delta R / R=1,4 \%$ are $16.5^{\circ}$ and $33^{\circ}$, respectively. For transparent interfaces, $k_{10}=k_{01}=0$, Eqs. (35) and (37) indicate that all phase changes are zero to second order in $\phi$. This is in agreement with the fact that all reflection phase shifts stay exactly constant up to the Brewster angle, with $\delta_{p}$ and $\Delta$ experiencing discontinuous jumps of $\pi$ at that angle. The phase changes at the air-gold interface in going from $\phi$ $=0$ to $\phi=15^{\circ}$ are $\Delta \delta_{s}=-\Delta \delta_{p}=1.66^{\circ}$ and $\Delta \Delta=-3.32^{\circ}$, as calculated from Eqs. (35) and (37), respectively.

It is possible to extend the second-order analysis to an arbitrary multilayer structure. A specific example of such an analysis, for high-reflectance multilayer dielectric mirrors, has been published previously. ${ }^{9}$

\section{ACKNOWLEDGMENT}

I am pleased to acknowledge support by the National Science Foundation under grant no. DMR-8018417.

\section{REFERENCES}

1. See, for example, D. L. Greenaway and G. Harbeke, Optical Properties and Band Structure of Semiconductors (Pergamon, New York, 1968), p. 25.

2. See, for example, M. Born and E. Wolf, Principles of Optics (Pergamon, New York, 1975), p. 38.

3. See, for example, R. M. A. Azzam and N. M. Bashara, Ellipsometry and Polarized Light (North-Holland, Amsterdam, 1977), p. 283.

4. R. M. A. Azzam, "AIDER: angle-of-incidence derivative ellipsometry and reflectometry," Opt. Commun. 16, 153-156 (1976).

5. V. M. Bermudez, "AIDER (angle-of-incidence derivative ellipsometry and reflectometry): implementation and application," Surf. Sci. 94, 29-40 (1980).

6. For such a proof, begin with the appropriate reflection coefficients, which are found, e.g., in Ref. 3, p. 354.

7. R. H. Muller, "Definitions and conventions in ellipsometry," Surf. Sci. 16, 14-33 (1969).

8. G. Hass, in Applied Optics and Optical Engineering, R. Kingslake, ed. (Academic, New York, 1965), Vol. III, Chap. 8, p. 316.

9. M. Flannery, E. Loh, Jr., and M. Sparks, "Nearly perfect multilayer dielectric reflectors: theory," Appl. Opt. 18, 1428-1435 (1979). 